\title{
COMA MIXEDEMATOSO
}

\author{
MYXEDEMA COMA
}

Léa M. Z. Maciel

Docente. Divisão de Endocrinologia. Departamento de Clinica Médica. Faculdade de Medicina de Ribeirão Preto - USP.

CORRESPONDÊNCIA: Av. Bandeirantes, 3900 - CEP 14048 - 900 - Ribeirão Preto - SP - FAX: (16) 6331144 e-mail: Imzmacie@fmrp.usp.br

MACIEL LMZ. Coma mixedematoso. Medicina, Ribeirão Preto, 36: 384-388, abr./dez. 2003.

RESUMO - O Coma Mixedematoso, manifestação extrema do estado hipotireóideo, representa uma situação clínica incomum, mas, potencialmente, letal. Pacientes com hipotireoidismo apresentam um série de adaptações fisiológicas para compensar a falta dos hormônios tireoidianos, porém em determinadas situações, como por exemplo, na vigência de uma infecção, não serão suficientes, o paciente descompensará e caminhará para o coma. Pacientes hipotireóideos, graves têm uma história longa de cansaço, ganho de peso, constipação intestinal e intolerância pelo frio. Na suspeita de coma mixedematoso, os pacientes deverão ser admitidos em unidade de terapia intensiva para cuidados respiratórios e cardiovasculares. A maioria dos pesquisadores recomenda o tratamento com levotiroxina (L-T4), ao invés da liotironina (T3), por via endovenosa. É de grande importância o reconhecimento da causa da descompensação e tratá-la agressivamente.

UNITERMOS - Coma Mixedema.

\section{1- CONCEITO}

Coma mixedematoso (CM) é uma condição clínica, rara, que se manifesta em pacientes com hipotireoidismo primário ou secundário, de longa duração, e não tratados. O termo "coma”, a rigor, é impróprio, pois, na maioria das vezes, o paciente não se apresenta comatoso. Talvez, o termo mais correto seria Crise Mixedematosa. Trata-se de emergência metabólica e cardiovascular que, quando não prontamente diagnosticada e tratada, está associada à mortalidade superior a $50 \%{ }^{(1,2)}$. É uma condição 4 a 8 vezes mais comum em mulheres do que em homens, o que se deve à maior incidência do hipotireoidismo primário no sexo feminino. O quadro pode ser precipitado por condições diversas, tais como: exposição ao frio, infecção, trauma, acidente vascular cerebral, insuficiência cardíaca (ICC), hemorragias gastrointestinais, hipoglicemia ou drogas (tranqüilizantes, sedativos, anestésicos, analgésicos, narcóticos, amiodarona, lítio, fenitoina) ${ }^{(3,45)}$.

\section{2- FISIOPATOLOGIA}

No hipotireoidismo de longa duração, algumas adaptações fisiológicas ocorrem para manter a homeostase, ainda que mediante um balanço precário. A redução do metabolismo basal e a diminuição do consumo de oxigênio resultam em vasoconstrição periférica para manter a temperatura corporal. O número de receptores $\beta$, adrenérgicos está reduzido, usualmente com preservação dos receptores a adrenérgicos e das concentrações de catecolaminas, levando a um desequilíbrio $\beta / \alpha$ adrenérgico, o que acarreta hipertensão diastólica e redução do volume sangüíneo total ${ }^{(6,7)}$.

O CM é uma forma de hipotireoidismo descompensado, na qual os mecanismos de adaptação não serão mais suficientes e, essencialmente, todos os órgãos são afetados.

Alterações neurológicas: não obstante os pacientes não se apresentem, na maioria das vezes, com quadro clínico de coma, alterações cognitivas 
importantes, como letargia, estupor, estão presentes em pacientes com CM. O mecanismo exato não é bem conhecido, mas a diminuição do fluxo cerebral, associada à diminuição do consumo de oxigênio e das concentrações de glicose, assim como a redução dos concentrações de T4 e T3 influenciam a função cerebral. Hiponatremia decorrente de disfunção renal pode ser um fator contribuinte adicional.

Alterações cardiovasculares: bradicardia e diminuição da contractilidade miocárdica determinam a diminuição do débito cardíaco ${ }^{(8)}$. Essas alterações são causadas por diminuição das proteínas contráteis dos miócitos e de enzimas, incluindo a $\mathrm{Na}^{+}, \mathrm{K}^{+}$ATPase, decorrentes da diminuição da transcrição gênica, na ausência de T3. Documenta-se aumento da resistência vascular periférica, que, embora dependente de múltiplos fatores, está associada à diminuição dos níveis de $\mathrm{T} 3^{(9)}$. $\mathrm{O}$ volume plasmático está reduzido; a aumentada permeabilidade capilar determina acúmulo de fluído nos tecidos e espaços e pode ser a causa do derrame pericárdico, encontrado em muitos pacientes ${ }^{(10)}$.

Alterações pulmonares: os pulmões, geralmente, não são afetados gravemente e a mais consistente anormalidade é a diminuição do controle ventilatório, acarretando, ambos, hipoxemia e hipercapnia ${ }^{(11,}$ 12). A alteração no controle ventilatório pode causar aumento de sensibilidade a drogas sedativas e causar depressão respiratória em um paciente com hipotireoidismo grave. A disfunção dos músculos respiratórios pode ocorrer. O acúmulo de fluido pode causar derrame pleural e diminuição da capacidade de difusão. A obesidade, se existente, causa diminuição do volume pulmonar, da capacidade de difusão e poderá ser a causa primária da hipoventilação, hipóxia, hipercapnia e depressão do controle respiratório, observadas nos pacientes com alterações pulmonares.

Alterações renais: a função renal poderá estar gravemente comprometida, em parte em decorrência do baixo débito cardíaco e da vasoconstrição, acarretando baixa taxa de filtração glomerular. Concentrações reduzidas de $\mathrm{Na}^{+}, \mathrm{K}^{+}$ATPase diminuem a reabsorção de sódio, assim como a excreção de água livre, resultando em hiponatremia ${ }^{(13)}$, que usualmente está presente no CM.

Alterações gastrointestinais: o hipotireoidismo com vários níveis de gravidade está associado à diminuição da motilidade intestinal. Nos pacientes com isso, poderá ocorrer atonia gástrica, megacólon ou íleo paralítico. Má absorção poderá, também, ser documentada. Ascite, apesar de incomum, pode se manifestar pelo aumento da permeabilidade capilar, insuficiência cardíaca ou outros mecanismos.

\section{3- QUADRO CLÍNICO}

Praticamente, todos os pacientes apresentam uma história longa de hipotireoidismo, com queixas de cansaço, intolerância ao frio, constipação intestinal e pele seca. Pacientes com tais manifestações podem progredir vagarosamente para letargia, delírio e coma ou apresentar rápida progressão do quadro, que se precipita por pneumonia ou outra infecção, exposição ao frio, ICC, acidente vascular cerebral, trauma ou superdosagem de medicações ${ }^{(14)}$.

Um conjunto de sinais clínicos podem ser encontrados e auxiliarão o diagnóstico. (Tabela I)

$\mathrm{Na}$ avaliação laboratorial, as principais alterações encontradas encontram-se na Tabela II.

\section{4- TRATAMENTO}

Se houver suspeita clínica de CM, o tratamento deverá ser iniciado imediatamente, mesmo antes da confirmação laboratorial. O paciente em CM corre risco de vida e deverá ser estabilizado em unidade de cuidados intensivos $^{(15)}$. As primeiras 24 - 48 horas são críticas.

\section{Prioridade Iniciais}

1. Ventilação mecânica, se houver hipercapnia/hipóxia significantes.

2. Reposição hormonal imediata, enquanto se aguardam resultados confirmatórios.

3. Aquecimento com cobertores comuns e uma sala quente (aquecimento rápido, ativo, é contra-indicado, pois pode provocar vasodilatação periférica e induzir o choque circulatório).

4. Administração de corticóides, após a coleta de sangue para cortisol plasmático.

5. Tratamento de infecção associada.

6. Correção de hiponatremia grave $(\mathrm{Na}<120 \mathrm{mEq} / \mathrm{L})$.

Outras intervenções incluem:

- correção de hipoglicemia com glicose, endovenosa (EV);

- tratamento de hipotensão grave, com administração cuidadosa de solução glicosada a 5-10\%, e solução fisiológica a $0,9 \%$ ou salina hipertônica, se existir hiponatremia $<120 \mathrm{mEq} / \mathrm{L}$ );

- reajuste de dose de qualquer medicação para compensar a diminuição da perfusão renal, ou o aumento da meia vida da droga, etc.;

- digital ou diuréticos deverão ser administrados com cautela para os pacientes com ICC.

Reposição Hormonal: como a absorção intestinal está comprometida, a terapia endovenosa está 


\section{Tabela I: Sinais clínicos em pacientes com Coma Mixedematoso}

Sinal

\begin{tabular}{|l|l|l|}
\hline Temperatura & $\begin{array}{l}\text { Hipotermia está usualmente presente. Caso a temperatura seja aferida com termômetro de } \\
\text { mercúrio, deve-se ter o cuidado ao posicionar o nível do mercúrio, caso contrário, a } \\
\text { temperatura do paciente poderá ser subestimada. A ocorrência de febre pode não ser } \\
\text { documentada em pacientes com infecção. }\end{array}$ \\
\hline Pulsos & \begin{tabular}{l} 
Frequiência e amplitude reduzidos \\
\hline Pressão arterial
\end{tabular} & Sistólica normal e diastólica elevada \\
\hline Cabelos & Grossos ou finos e ralos \\
\hline Facies & Grotesco com edema facial ou periorbitário, macroglossia \\
\hline Tireóide: & $\begin{array}{l}\text { Aumentada de volume ou, mesmo, não palpável. Presença de cicatriz por cirurgia anterior } \\
\text { pode ser observada }\end{array}$ \\
\hline Pulmões & $\begin{array}{l}\text { Freqüência respiratória baixa, sinais de congestão pulmonar, derrame pleural, consolidação } \\
\text { no parênquima pulmonar }\end{array}$ \\
\hline Abdome & $\begin{array}{l}\text { Distensão secundária ao îleo paralítico e/ou ascite, diminuição ou ausência de ruídos } \\
\text { intestinais }\end{array}$ \\
\hline Coração & $\begin{array}{l}\text { Bradicardia, diminuição do impulso apical, bulhas cardíacas abafadas, aumento da área } \\
\text { cardíaca, derrame pericárdio }\end{array}$ \\
\hline Extremidades & Frias, com edema não depressível em mãos e pés \\
\hline Pele/unhas & Espessada; seca; amarelada (devido à hipercarotenemia); unhas quebradiças \\
\hline Neuromuscular & $\begin{array}{l}\text { Confusão, torpor, obnubilação, coma, fala lenta, convulsões, reflexos com fase de } \\
\text { relaxamento lenta }\end{array}$ \\
\hline
\end{tabular}

indicada. O uso de $\mathrm{T} 4$ apenas, ou da combinação de $\mathrm{T} 4$ e T3, ou de T3 apenas permanece controverso ${ }^{(13,17)}$. A conversão de T4 para o T3, nesses pacientes, está prejudicada. Assim, a administração de T3 seria recomendável. Entretanto, a administração de T3, por ser de ação imediata e ter meia-vida curta, pode, com maior probabilidade, provocar arritmias, principalmente se a função miocárdica estiver comprometida.

Dose recomendada: dose de ataque: 200 - 500 mcg de Levotiroxina (L-T4), EV. Seguida de doses diárias de 50 - $100 \mathrm{mcg}$, EV. Quando o paciente estiver se alimentando, a L-T4 deverá ser administrada, por via oral, na dose de $50-100 \mathrm{mcg} / \mathrm{dia}$. Doses menores são recomendadas para o paciente idoso ou quando isquemia miocárdica for suspeitada. Alguns pesquisadores recomendam dose adicional de T3, EV, para pacientes jovens e com baixo risco cardiovascular ou quando não houver nenhuma melhora clínica nas primeiras 24 a $48 \mathrm{~h}$. A dose indicada é de $10 \mathrm{mcg}$ a cada 4 h ou 25 mcg a cada 8 h, por via EV.

Terapia com esteróides: Hidrocortisona - 100 mg a cada $8 \mathrm{~h}, \mathrm{EV}$, continuadamente até que o a valor da dosagem de cortisol plasmático, colhido previamente, seja conhecido e mostre-se normal.

Infecção: freqüentemente o evento precipitante do coma mixedematoso é uma infecção oculta ou declarada. Febre ou elevação de leucócitos, usualmente, estão ausentes, embora desvio à esquerda seja observado freqüentemente. Deve-se obter culturas de diversos fluidos e iniciar tratamento empírico com antibióticos de amplo espectro e, posteriormente, tal tratamento deve ser reavaliado, se a causa da infecção for identificada.

Isquemia miocárdica: pode ser o fator precipitante do CM no paciente idoso ou poderá ocorrer subseqüentemente. A dosagem da creatina fosfoquinase (CPK) com suas frações poderá ajudar no diagnóstico e tratamento de um evento coronariano agu- 
Tabela II: Exames a serem solicitados em pacientes com coma mixedematoso e os resultados esperados.

\begin{tabular}{l|l|l|l|l}
\hline Exames & $\begin{array}{l}\text { Geralmente elevado, indicando uma doença tireoidiana primária, em caso de } \\
\text { concentraçoses normais ou baixas, deverá ser considerado um hipotireoidismo } \\
\text { secundário, a não ser que uma doença grave e/ou drogas tais como: dopamina ou } \\
\text { glicorticóides, que causam supressão de TSH, estejam sendo utilizadas }\end{array}$ \\
\hline T4 livre & Baixo ou indetectável \\
\hline Eletrólitos & Hiponatremia comumente encontrada \\
\hline Osmolaridade sérica & Baixa \\
\hline Creatinina sérica & Usualmente, elevada devido à diminuição da perfusão renal \\
\hline Glicemia & Hipoglicemia é comum, mas poderá também sugerir insuficiência adrenal \\
\hline Creatina quinase (CK) & Freqüentemente elevada \\
\hline Hematócrito & Geralmente < 30\% \\
\hline Gasometria arterial & Aumento da PCO2 e diminuição da PO2 \\
\hline Cortisol & Deverá ser determinado antes do início da terapia com esteróides endovenosos \\
\hline Eletrocardiograma & $\begin{array}{l}\text { Bradicardia sinusal, complexos QRS de baixa amplitude, QT prolongado, ondas T } \\
\text { achatadas ou invertidas }\end{array}$ \\
\hline RX de tórax & Cardiomegalia, derrame pleural \\
\hline
\end{tabular}

do, porém, é importante estar ciente de que as concentrações da CPK, freqüientemente, estão elevadas no coma mixedematoso ${ }^{(17,18)}$.

Caso a isquemia ou o infarto sejam diagnosticados, ou mesmo se o paciente tiver fatores de risco significantes para doença coronariana, a terapia hormonal tireoidiana deverá ser feita em dose menores.

\section{Reposição de volume}

Administração de glicose e de salina deverá ser realizada cuidadosamente, porque os pacientes são, usualmente, hipervolêmicos e podem desenvolver ICC em face da disfunção ventricular, decorrente do hipotireoidismo. Se o $\mathrm{Na}$ for $<120 \mathrm{mEq} / \mathrm{l}$, considerar a administração de salina hipertônica, EV, seguida de furosemide, EV.

Quando ocorrer hipotensão, que não melhora com a reposição de líquidos, transfusão sangüínea deverá ser considerada. Finalmente, administração cuidadosa de Dopamina poderá ser utilizada. Geralmente, a hipotensão é resistente às drogas usuais até que hormônios tireoidianos e glicocorticóides sejam administrados em doses adequadas.

\section{Tratamento cirúrgico}

Os pacientes deverão ser estabilizados com T4 e glicocorticóides antes de procedimentos cirúrgicos. Em situações que requerem cirurgia de urgência, deverá ser administrada uma dose de 200-500 mcg de L-T4 e glicocorticóides, endovenosos, antes da indução anestésica. Monitorização cardiovascular com cateter de Swan-Ganz é requerida.

\section{Dieta}

Considerando que a motilidade intestinal está usualmente diminuída, a ingestão alimentar não deverá ser permitida até que o paciente esteja alerta e com ruídos hidroaéreos ativos, quando, então, alimentação pastosa deverá ser gradualmente instituída.

São considerados fatores de mau prognóstico: temperatura corporal abaixo de $34^{\circ} \mathrm{C}$; hipotermia persistente, não responsiva até $72 \mathrm{~h}$ de terapia; idade avançada; bradicardia com freqüência cardíaca menor que 44 bp/min; septicemia; infarto do miocárdio e hipotensão arterial. 
MACIEL LMZ. Myxedema coma. Medicina, Ribeirão Preto, 36: 384-388, apr./dec. 2003

ABSTRACT - Myxedema coma, the extreme manifestation of hypothyroidism, is a uncommon but potentially lethal condition. Patients with hypothyroidism may exhibit a number of physiologic alterations to compensate for the lack of thyroid hormone. If these homeostatic mechanisms are overwhelmed by factors, such as infection, the patient may decompensate into a myxedema coma. Patients with hypothyroidism typically have a history of fatigue, weight gain, constipation and cold intolerance. Patients with suspected myxedema coma should be admitted to an intensive care unit for vigorous pulmonary and cardiovascular support. Most authorities recommend treatment with intravenous levothyroxine (T4) as opposed to intravenous liothyronine (T3). It is always important to search diligently to determine the underlying cause of the decompensation and to treat that aggressively.

UNITERMS - Myxedema Coma.

\section{REFERÊNCIAS BIBLIOGRÁFICAS}

1 - NICKERSON JF; HILL SR; MCNEIL JH \& BAKER SB. Fatal myxedema, with or without coma. Ann Intern Med 53: 475493, 1960.

2 - FORESTER CA. Coma in myxedema. Report of a case and review of the world literature. Arch Intern Med 111: 100109, 1963.

3 - HYLANDER B \& ROSENQVIST U. Treatment of myxoedema coma-factors associated with fatal outcome. Acta Endocrinol (Copenhagem) 108: 65-71, 1985.

4 - SANTIAGO R \& RASHKIN MC. Lithium toxicity and myxedema coma in an elderly woman. J Emerg Med 8: 63-66, 1990.

5 - MAZONSON PD; WILLIAMS ML; CANTLEY LK; DALLDORF FG; UTIGER RD \& FOSTER JR. Myxedema coma during longterm amiodarone therapy. Am J Med 77: 751-754, 1984.

6 - PARK CW; SHIN YS; AHN SJ; KIM SY; CHOI EJ; CHANG YS \& BANG BK. Thyroxine treatment induces upregulation of renin-angiotensin-aldosterone system due to decreasing effective plasma volume in patients with primary myxoedema. Nephrol Dial Transplant 16:1799-1806, 2001.

7 - VILLABONA C; SAHUN M; ROCA M; MORA J; GOMEZ N GOMEZ JM; PUCHAL R \& SOLER J. Blood volumes and renal function in overt and subclinical primary hypothyroidism. Am J Med Sci 318: 277-280, 1999.

8 - KLEIN I \& OJAMAA K. The cardiovascular system in hypothyroidism. In: BRAVERMAN LE \& UTIGER RD, eds. The thyroid: A fundamental and clinical text. $7^{\text {th }}$ ed, Lippincott Williams and Wilkins, Philadelphia, p. 799-804, 2000.

9 - DIEKMAN MJ; HARMS MP; ENDERT E;WIELING W \& WIERSINGA WM. Endocrine factors related to changes in total peripheral vascular resistance after treatment of throtoxic and hypothyroid patients. Eur J Endocrinol 144: 339-346, 2001.
10 - RACHID A; CAUM LC; TRENTINI AP; FISCHER CA;ANTONELLI DA \& HAGEMANN RP. Pericardial effusion with cardiac tamponade as a form of presentation of primary hypothyroidism. Arq Bras Cardiol 78:580-585, 2002.

11 - DOMM BM \& VASSALLO CL. Myxedema coma with respiratory failure. Rev Respir Dis 107: 842-845, 1973.

12 - ZWILLICH CW; PIERSON DJ; HOFELDT FD; LUFKIN EG \& WEIL JV. Ventilatory control in myxedema and hypothyroidism. N Engl J Med 292: 662-665, 1975.

13 - JORDAN RM. Myxedema coma. Pathophysiology, therapy and factors affecting prognosis. Med Clin North Am 79: 185-194, 1995

14 - GICK GG; ISMAIL-BEIGI F \& EDELMAN IS. Thyroidal regulation of rat renal and hepatic Na,K-ATPase gene expression. J Biol Chem 263: 16610-16618, 1988.

15 - RINGEL MD. Management of hypothyroidism and hyperthyroidism in the intensive care unit. Crit Care Clin 17: 59-74, 2001.

16 - WARTOSKY L. Myxedema coma In: WERNER SC, INGBAR SH, BRAVERMAN LE \& UTIGER RD, eds. The thyroid: A fundamental and clinical text. $7^{\text {th }}$ ed, Lipincott Williams \& Wilkins, Philadelphia, p. 871-877, 2000.

17 - NEE PA; SCANE AC; LAVELLE PH; FELLOWS IW \& HILL PG Hypothermic myxedema coma erroneously diagnosed as myocardial infarction because of increased creatine kinase MB. Clin Chem 33: 1083-1084, 1987.

18 - HICKMAN PE; SILVESTER W; MUSK AA; MCLELLAN GH \& HARRIS A. Cardiac enzyme changes in myxedema coma. Clin Chem 33: 622-664, 1987. 